\title{
Dislocation bias factors in fcc copper derived from atomistic calculations
}

\author{
Zhongwen Chang ${ }^{\mathrm{a}}$, Pär Olsson ${ }^{\mathrm{a}}$, Dmitry Terentyev ${ }^{\mathrm{b}}$, Nils Sandbergc,a \\ ${ }^{a}$ KTH Royal Institute of Technology, Reactor Physics, Roslagstullsbacken 21, SE-10691 \\ Stockholm, Sweden \\ ${ }^{b}$ SCK-CEN, Nuclear Materials Science Institute, Boeretang 200, B-2400 Mol, Belgium \\ ${ }^{c}$ Swedish Radiation Safety Authority, Solna Strandväg 96, SE-171 16 Stockholm, Sweden
}

\begin{abstract}
Atomistic calculations were employed in order to calculate the interaction energy of an edge dislocation with different point defects. The bias factor was calculated by applying a finite element method on the interaction energy landscapes obtained from the atomistic calculations. A comparison of the calculated bias factor with a model based on elasticity theory reveals around 30\% discrepancy under conditions representative for electron irradiation at $600^{\circ} \mathrm{C}$. Possible reasons are discussed. The bias factor dependence on dislocation density and ambient temperature is presented and discussed. Keywords: Dislocation bias, Atomistic calculation, Interaction energy
\end{abstract}

1. Introduction

Austenitic steels are used in nuclear reactors as structural materials since they have excellent mechanical properties. Swelling under irradiation, how-

Email addresses: zhongwen@kth.se (Zhongwen Chang), polsson@kth.se (Pär Olsson) 
4 ever, is a significant drawback that is especially pronounced in austenitic 5 steels [? ]. The swelling of structural materials limits the lifetime of internal 6 reactor components and also the maximal fuel burn up [? ]. Therefore, great 7 efforts have been devoted to improve the swelling resistance of austenitic steels [? ? ? ].

$9 \quad$ The 316 type stainless steel (SS316) is a typical austenitic steel that is widely used for internal parts of light water reactors and has therefore been

comprehensively studied. Improvements of SS316 led to the development of 15-15Ti, which today is one of the best performing steels for nuclear reactor applications [? ? ? ]. Its incubation dose for swelling is greater than 100 dpa $[?]$. The current design of fast reactors, however, requires resistance to even higher doses. In the ASTRID sodium fast reactor, the cladding material is expected to work for 8-10 years under temperatures above $700 \mathrm{~K}$ and accumulate an irradiation dose of over 150 dpa [? ]. Should a new, even more durable steel be developed, it is of use to have an improved fundamental understanding of the mechanisms of irradiation swelling.

Austenitic steels are face-centered cubic (fcc) alloys and in order to address phenomena in such an alloy, it is convenient to begin by modeling an fcc metal. Given the fact that nickel is the fcc stabilizing element in most austenitic alloys, one would preferably use it as a model metal. Nickel, however, has not been extensively studied and there is a scarcity of experimental data from electron irradiation. Copper, another fcc metal, on the other hand, boasts a large database of irradiation experiments and is thus more suitable 
for computational modeling of irradiation induced swelling. In addition, the stacking-fault energy (SFE) plays an important role for dislocation properties. Copper has an SFE rather close to that of austenitic steels, while the SFE of nickel is much higher.

One of the most popular models used for studies of dimensional changes due to irradiation, is the standard rate theory (SRT) [? ? ? ]. It is formulated within the framework of the mean field type chemical reaction rate theory. In this model, irradiation damage produces only Frenkel pairs and they are created randomly in space and time. The concept of sink bias is introduced in the model. One example of the sink bias, is the dislocation bias $\left(B_{\mathrm{d}}\right)$, which implies different absorption rates of interstitials and vacancies at sinks [? ]. Another example is void bias, which is significantly smaller compared to the dislocation bias [? ]. For simplicity, the void bias is neglected in the standard rate theory model [?], which implies that the dislocation bias becomes the only driving force for micro structure evolution in the model. The swelling rate at steady state depends on density and size of voids and on dislocation bias.

The description of defect production in this model is accurate only for the case when the energy of the injected particles is close to the displacement threshold [? ]. Neutron irradiation generates recoils with much higher energy hence it induces clusters of point defects formed directly in cascades. Therefore, the simplified SRT model is not suitable for modeling the effects of neutron irradiation. For that purpose, a more sophisticated model like 
the production bias (PBM) [? ? ? ] model has been developed. Still, the dislocation bias factor is still an integral part of the model.

A large number of experiments [? ? ? ] and theoretical studies [? ? ? ? ] have been performed in order to assess factors controlling the bias. In these studies, the SRT model was applied to fit the experimental data in order to obtain $B_{\mathrm{d}}[?]$ and elasticity theory was used to derive the analytical expression of sink strengths. The validity of such approaches can, however, be questioned. The analytical interaction from elasticity theory, which is derived from the first order size interaction, is not valid in the vicinity of the dislocation core due to the mathematical divergence in approaching the core. Furthermore, it has been long recognized that theoretically estimated values of the dislocation bias generally exceed those based on fitting to experimental data. This indicates that the role of dislocation - point defect (PD) interaction is not completely understood. In general, all calculations of the bias factor rely on an elastic description of the dislocation - point defect interaction. This would be sufficient if the dominating energy term ruling the capture process stems from the interaction far from dislocation core. On the other hand, the detailed processes of point defect diffusion close to the core will at some point be of importance. It is therefore natural to ask if deviations from elasticity theory and atomistic effects are important in determining the dislocation bias. Edge dislocation - point defect interaction energies have previously been calculated in $\mathrm{Fe}$ and $\mathrm{Ni}$ using an atomistic approach[? ]. In $\mathrm{Cu}$ and Mo elasticity theory has been applied in order to 
calculate the point defect interaction energy close to the dislocation core[? ]. However, in these studies, the interaction energy maps were not used to obtain estimates of sink strengths or the dislocation bias.

In the present work, large scale atomistic simulations with empirical potentials were applied in order to map the dislocation - point defect interaction energy in an fcc copper lattice. Furthermore, the results were used in order to derive the dislocation bias and the swelling rate as a function of temperature and dislocation density. Thus the importance of the core region of the dislocation is addressed in terms of determining the bias and ultimately the swelling rate. More precisely, the ranges of temperatures and dislocation densities in which the core region is of importance are discussed. Consequently, the ranges in which it can be neglected are deduced.

\section{Theory and Methods}

\subsection{Bias factor}

The main assumptions of the simplest dislocation bias model are as follows [? ? ]: 1) the incident particles only produce isolated Frenkel pairs (FPs), 2) both self-interstital atoms (SIAs) and vacancies migrate in three dimensions, and 3) dislocations preferentially absorb SIAs, rather than vacancies, due to the stronger dislocation-SIA interaction. In this model, dislocations are the only sinks with a bias factor different than unity, and consequently the only driving force for the evolution of the micro-structure after electron irradiation. The dislocation bias is then the reason for the excess of vacancies in 


$$
\frac{d S}{d \phi}=k_{\mathrm{v}}^{2}\left(Z_{\mathrm{v}}^{\mathrm{v}} D_{\mathrm{v}} C_{\mathrm{v}}-Z_{\mathrm{i}}^{\mathrm{v}} D_{\mathrm{i}} C_{\mathrm{i}}\right) \approx B_{\mathrm{d}} \frac{k_{\mathrm{c}}^{2} Z_{\mathrm{v}}^{\mathrm{d}} \rho_{\mathrm{d}}}{\left(k_{\mathrm{c}}^{2}+Z_{\mathrm{v}}^{\mathrm{d}} \rho_{\mathrm{d}}\right)\left(k_{\mathrm{c}}^{2}+Z_{\mathrm{i}}^{\mathrm{d}} \rho_{\mathrm{d}}\right)}
$$

97 where $k_{\mathrm{c}}^{2}=4 \pi\langle R\rangle N$ and $B_{\mathrm{d}}=\frac{Z_{\mathrm{i}}^{\mathrm{d}}-Z_{\mathrm{v}}^{\mathrm{d}}}{Z_{\mathrm{v}}^{\mathrm{d}}}$ with $\phi$ the irradiation dose in dpa,

the bulk and consequently for void swelling. The swelling rate is hence given by $[?]$ : $k_{\mathrm{v}}^{2}$ the sink strength of voids, $Z^{\mathrm{d}}$ the sink strength of dislocations (sometimes referred as capture efficiency), $D$ the diffusion coefficient, and $C$ the point defect concentration. The subscripts i and v represent interstitial and vacancy respectively and superscripts $\mathrm{v}$ and $\mathrm{d}$ mean void and dislocation, respectively. $B_{\mathrm{d}}$ is the dislocation bias factor, $\rho_{\mathrm{d}}$ is the dislocation density, $\langle R\rangle$ and $N$ are the mean void radius and the void number density. The approximation in the equation assumes that the capture efficiency of voids is the same for both vacancy and interstitial, i.e. $Z_{\mathrm{v}}^{\mathrm{v}}=Z_{\mathrm{i}}^{\mathrm{v}}$.

The distortions caused by the formation of defects in crystal structure are described as stress fields in elasticity theory. The interactions of different defects originate from the overlap of stress fields. The flux of point defects due to diffusion and to the stress field interaction can be described by Fick's law including a drift term:

$$
J=-\nabla(D C)-\beta D C \nabla E
$$

111 where $E$ is the interaction energy of dislocations with point defects. In order to solve this equation, it is most convenient to reformulate it as: 


$$
J=-e^{-\beta E(r, \theta)} \nabla \Psi
$$

where $\Psi=D C e^{\beta E(r, \theta)}$.

With the steady state condition $\nabla J=0$, Eq.2 is reduced to the form:

$$
\nabla^{2} \Psi=\beta \nabla E \cdot \nabla \Psi
$$

The assumption is that at the dislocation core, all point defects are absorbed. Hence the boundary condition at the dislocation core $r=r_{0}$, is $\Psi_{r_{0}}=0$. At the external boundary, i.e. the dislocation radius of influence, $r=R$, the defect concentration $C(r, \theta)$ is a constant and the interactions vanish. Hence, $\Psi_{R}=C^{\text {eq }}$ where $C^{\text {eq }}$ is the concentration of point defects in the steady state.

Eq.4 is numerically solved by applying the finite element method, which is encoded in the MATLAB PDEtoolbox. The sink strengths and the bias factor were obtained by integrating the total flux around the dislocation core. The sink strength is defined as the ratio of PD fluxes with and without interaction with the dislocation, i.e. $Z=\frac{J}{J_{0}} . J$ is the flux of PDs including the interaction with the dislocation and $J_{0}$ is the flux excluding the interaction.

The effect of the FEM geometry on the sink strengths and the bias factor was investigated using different inner absorption radii $r_{0}$. The absorption boundary was applied either as a single circle around the two partial cores, or as two separate, touching circles, one around each core. For a given 
absorption length, equal to the total circumference of the core absorption boundaries, the effect of choosing one or two boundaries was seen to be small, the difference varies from $0.2 \%$ to $6 \%$ depending on the temperature. The data here presented has the single core configuration with the inner radius of $38 \AA$, which is the same as the splitting distance.

\subsection{Atomistic calculations}

In Eq.4 the interaction energy is an important input parameter. The analytical expression for the interaction energy in elasticity theory has been derived from the first order size interaction of dislocations and point defects by treating the medium as being elastically isotropic [? ]:

$$
E=-A \frac{\sin \theta}{r}
$$

where

$$
A=\frac{\mu b}{3 \pi} \frac{1+\nu}{1-\nu}|\Delta|
$$

in polar coordinates $(r, \theta) . \mu$ is the shear modulus, $\nu$ is Poisson's ratio, $b$ is the Burgers vector, and, $\Delta$ is the dilatation volume of the PD.

To obtain the atomistic interaction, a model treating a periodic array of edge dislocations was applied. Two half crystals are strained to have different lattice parameters in the $\langle 110\rangle$ direction, along the Burgers vector $b$. They join along the dislocation slip plane to form a misfit of $b$. More extensive details can be found in a dedicated study [? ].

In order to model an infinite straight edge dislocation (ED), periodic 
boundary conditions were applied in the direction of the Burgers vector $b$ and in the direction of the dislocation line. A fixed boundary condition was applied normal to the glide plane. A vacancy is created by removing one atom from the lattice and relaxing the crystal. Dumbbell SIAs in $\langle 100\rangle$ directions are created by adding one atom, perturbing slightly the atomic positions of the two nearest neighbor atoms and then relaxing. An initial distance of $0.4 a_{0}$ between the two relocated atoms is used. Considering both the boundary effects and the computational cost, a simulation box of 85980 atoms with the box dimensions of $99 b \times 10.4 b \times 58.8 b$ in the [110], [1112]], [1111] directions, was set up. This gives a dislocation density of $2.6 \cdot 10^{15} \mathrm{~m}^{-2}$. A combination of conjugate gradient and quasi static relaxation with constant volume was applied in the ideal dislocation structure.

Large scale molecular statics calculations were performed using the DYMOKA code [? ]. Full interaction energy landscapes around the dislocation core for PDs were obtained using a semi-empirical embedded atom method (EAM) potential for $\mathrm{Cu}[?]$. This potential reproduces the properties of defects in good agreement with experimental data. The stacking fault energy obtained from the potential is $44 \mathrm{~mJ} / \mathrm{m}^{2}$, the vacancy dilatation volume is $-0.3 \Omega$ and the interstitial dilatation volume is $1.8 \Omega$, where $\Omega$ is the atomic volume.

Different dislocation densities were generated by expanding the region described by the atomistic model and matching it to the analytical solution in the outskirts. In this manner the near core region is described as accurately as possible while at the same time one can obtain dislocation densities on 
the same order of magnitude as in technological materials. The effect of the fixed boundary and that of the periodic image interaction along the glide direction were corrected for in the atomistic simulation.

\section{Results and discussion}

\subsection{Interaction energy}

After the relaxation of a perfect dislocation, a splitting distance of 38 $\AA$ was obtained for the partial dislocation cores. The interaction energies of SIAs and vacancies with the dislocation are depicted in Fig.1 both from the atomistic model and the elastic model. The SIA interaction map is produced by averaging the results for three different dumbbell orientations, namely [100], [010] and [001]. Given that the original analytical expression is derived from a single core dislocation, a model of two partial cores, each with a Burgers vector $b / 2$ has been constructed. The superposition of the interaction energy Eq.5 due to the two cores forms the analytical elastic interaction model.

An assessment of the interaction between an SIA and the dislocation core reveals a significant discrepancy between the analytical solution, treating SIAs as isotropic objects, and results of the atomistic calculations, as can be seen in Fig.1. The atomistic description of the SIA-dislocation interaction in the core and stacking fault (binding the two partial cores) regions is essential given the anisotropy of $\mathrm{Cu}$, considered here, and for metals in general. 
Moreover, in the attractive side of the dislocation, in the case of interstitials, the interaction is stronger in the atomistic case compared to the analytical expression. Qualitatively, this can be understood because it is energetically easier to expand the lattice than to compress it far beyond the linear regime. For the vacancy, the difference is generally smaller. This leads to a larger dislocation bias in comparison with the analytical results.

\subsection{Bias factor}

By applying the atomistic interaction energy maps in Eq.4, numerical solutions of $\Psi$ were obtained on the dislocation plane. The calculated sink strengths and bias factor without the expansion at $873 \mathrm{~K}$ are demonstrated in Tab.1. The major difference in sink strength and bias factor, of about $30 \%$, from the two approaches stems from the difference in the SIA-dislocation interactions as shown in Fig.1.

Although the crystal size, used in the molecular static calculations, is large enough to accurately describe the dislocation core region, the implied dislocation density is 2-3 orders of magnitude higher than the one typical for real steels. By merging the energy landscape near the dislocation core, obtained through the atomistic simulation, with the elastic solution far away from the core, which has been proven to be accurate enough, the effect of the dislocation density on the value of $B_{\mathrm{d}}$ is characterized. It is shown in Fig.4 that $B_{\mathrm{d}}$ rises steeply if dislocation density exceeds $10^{14} \mathrm{~m}^{-2}$. Such a dislocation density can be reached by, for example, cold work. However, in 
service conditions at elevated temperatures, recovery would occur to lower the dislocation density. Nevertheless, neutron irradiation generates dislocation loops whose contribution to the total dislocation density at a certain dose becomes comparable and then even higher than the initial dislocation density. Importantly, dislocation loops contain a different type of Burgers vector (i.e. $\langle 100\rangle$ in bcc Fe-based steels and $1 / 3\langle 111\rangle$ in fcc FeNiCr-based steels) and character than the pre-existing dislocations. That is, most of the observed irradiation induced loops are of edge type. Whereas initial dislocations are usually of screw character with $b=1 / 2\langle 111\rangle$ in bcc and $1 / 6\langle 112\rangle$ in fcc metals. Hence, it is essential to make an appropriate evaluation of $B_{\mathrm{d}}$ for dislocation loops, since they become the predominant objects controlling the swelling process.

To assess to which extent the superposition of the analytical interaction would be in line with the correct description of a split dislocation core, $B_{\mathrm{d}}$ is calculated for different configurations. Fig.2 shows the results. The fully analytical solution, which depends only on the geometry of the dislocation system and the dilatation volumes of the point defects, is presented by Dubinsko et al [? ]. This analytical solution of the sink strength has approximated boundary conditions. The approximation that interactions on the outer boundary is negligible is not valid for high dislocation densities. The FEM approach overestimates the $B_{\mathrm{d}}$ by $1 \%$ for densities lower than $10^{14}$ $\mathrm{m}^{-2}$. The geometrical shape of the outer boundary does not seem to play a role, while the core configuration, namely one single full core or two partial 
half cores, does make a difference of around $0.5 \%$ in $B_{\mathrm{d}}$ values. Thus the superposed analytical model represents the essence of the original analytical model.

Sink strengths were calculated under different temperatures and dislocation densities by applying both the atomistic interaction energy and the elastic analytical interaction formula. The results are shown in Fig.3 and Fig.4. Both the atomistic and the analytical $B_{\mathrm{d}}$ decrease as the temperature increases. They tend to converge at the high temperature limit. The reason for that could be the contribution from the dislocation cores. As temperature increases, diffusion and thermal fluctuations become the dominant driving force for the flow of PDs. Therefore, in Eq.2, the contribution from the drift term decreases. The dislocation core region does not play as important a role as it does at lower temperatures. Thus the atomistic and analytical approaches converge in the high temperature limit. On the contrary, at low temperatures, segregation and nucleation plays an important role for the bias, hence the simplified model does not apply for an accurate bias calculation.

It worth noticing that a strong temperature dependence of $B_{\mathrm{d}}$ is demonstrated, which is important to account for when considering real structural or functional reactor components, which exhibit strong temperature gradients upon operation.

Fig.4 shows that $B_{\mathrm{d}}$ increases as the dislocation density increases. At higher temperatures, $B_{\mathrm{d}}$ is more weakly dependent on dislocation density 
than at lower temperatures. The atomistic model, again, has a higher derivative than the analytical model. At the low density limit, the calculated bias factor from atomistic and analytical interaction energy models tend to converge. For high dislocation densities the difference is significant. The possible reasons are that, with low dislocation density, diffusion of point defects are prioritized. On the other hand, at high dislocation densities, the dislocation core - point defect interactions are dominating, hence the precise core description becomes more important in this case. In fact, in real fast reactor conditions, high irradiation doses generates high dislocation densities. Therefore, the high density $B_{\mathrm{d}}$ results are of technological interest. The approach which has been developed here can readily be applied to dislocation loops, which have high generation rates in cascade inducing fast neutron spectra. In order to compare these results with measurements, experimental void density and void size data were selected. Together with the sink strengths obtained from the atomistic approach, the swelling rate is calculated in three different irradiation dose conditions. The experimental data is from Singh et al. [? ]. The electron- and proton irradiation data were selected for the reason that the fraction of defects produced in clustered configurations in those cases were sufficiently small for the dislocation bias model to be valid. In Table.2 the reference swelling is the one obtained from experiments [? ]. The calculated swelling is obtained by applying the sink strengths from the atomistic calculation to the SRT model at the same temperature and dislocation density as in the experiments. The discrepancies are within 
expected range considering that the SRT model here applied is a simple steady state model while transient period has been taken into account in the experimental fitting. Furthermore, the mean radius of voids is used here while in experimental fitting the distribution of voids sizes are used.

Due to the feasibility of combining atomistic calculations with numerical solutions of the diffusion equations, the applied method here can also be used in order to estimate the bias not only for dislocation-like objects, but in general for other objects that are considered as sinks for point defects, such as grain boundaries or non-coherent precipitates (e.g. ODS particles). Moreover, in technological steels, impurity segregation to the dislocation core can further modify the interaction energy landscape. The atomistic approach, as applied here, can readily be a solution to evaluate the impact of segregation effects on $B_{\mathrm{d}}$, given an appropriate cohesive model.

\section{Conclusions}

In this work, a atomistic calculation of the edge dislocation - point defect interaction energy map in fcc copper was performed and applied to calculate the bias factor. A combined method, of atomistic calculations imposed on a finite element method based numerical solution of Fick's law with a drift term, was elaborated in order to determine the sink strengths of the dislocation. In general, the sink strength is higher for interstitials than for vacancies. The same result applies for the interaction energies, that is, interstitials have higher interaction energies with the dislocation than vacancies 
do. Furthermore, the bias factor, as well as the interaction energies, obtained from the atomistic approach are compared with the ones obtained using an analytical model derived from elasticity theory. The dislocation bias predicted by the atomistic approach is higher than that predicted by the elastic approach, especially for high dislocation densities. This implies that an atomistic description of the dislocation - point defect interaction in fcc materials is important for high dislocation densities. It is also shown that the deviation, with respect to the atomistic approach, of the interaction energies obtained from elasticity theory is larger for interstitials than for vacancies. Furthermore, the strongest deviation is at the attractive (tensile) side of the dislocation in the case of the interstitials. This is the reason the dislocation bias factor predicted by the atomistic approach is higher than that predicted by elasticity theory. 


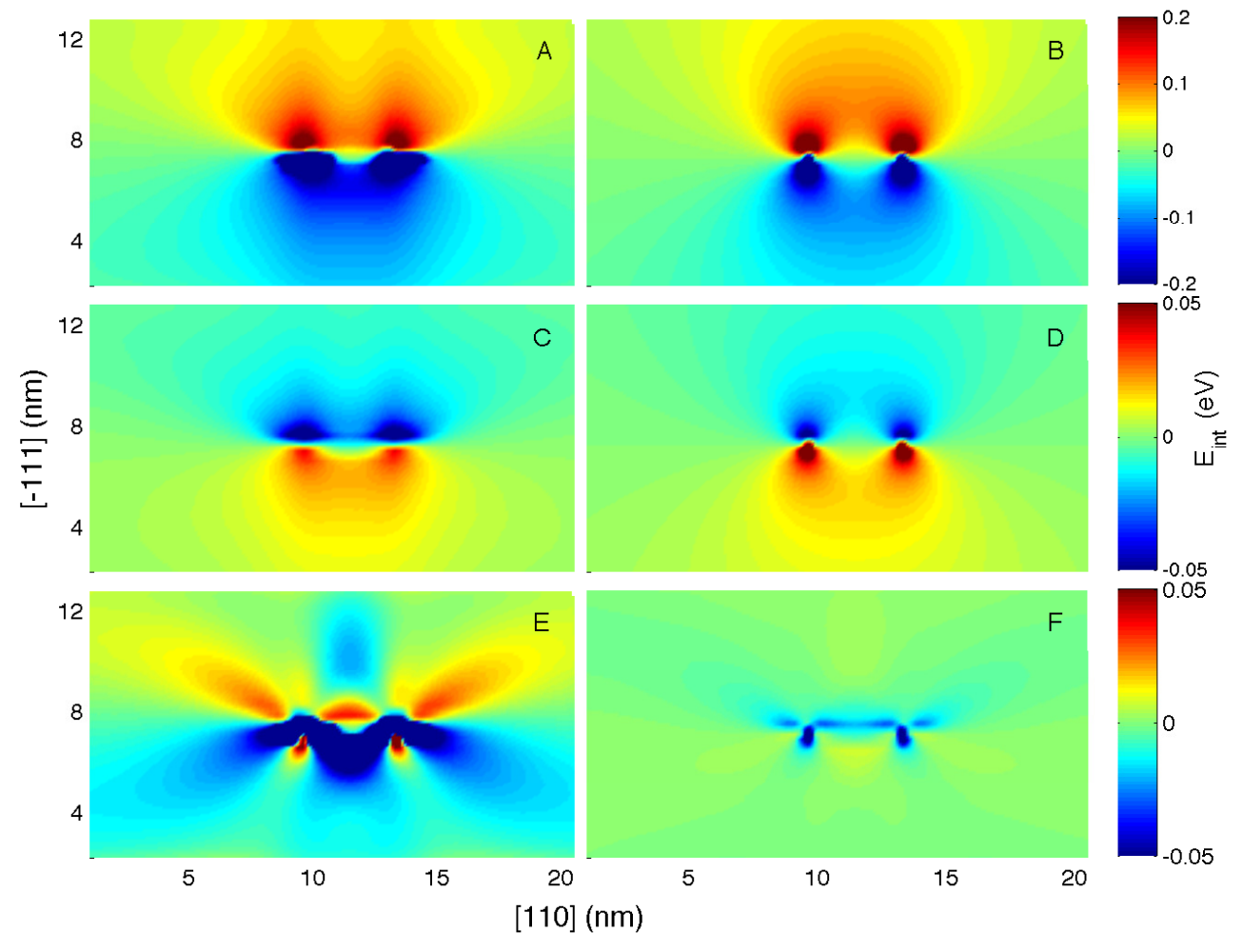

Figure 1: Edge dislocation - point defect interaction energy maps for the different approaches, A) Atomistic SIA; B) Analytical SIA; C) Atomistic vacancy; D) Analytical vacancy; E) Difference between A and B; F) Difference between $\mathrm{C}$ and $\mathrm{D}$. 




Figure 2: Analytical solution of $B_{\mathrm{d}}$ at $873 \mathrm{~K}$ as a function on dislocation density considering different geometries and methods. Illustration of different boundaries are shown in inner figures. A. one core with circle boundary; B. two cores with square boundary; C. one core with square boundary. Red circles represent the inner core, black circle and squares represent the outer boundaries. 


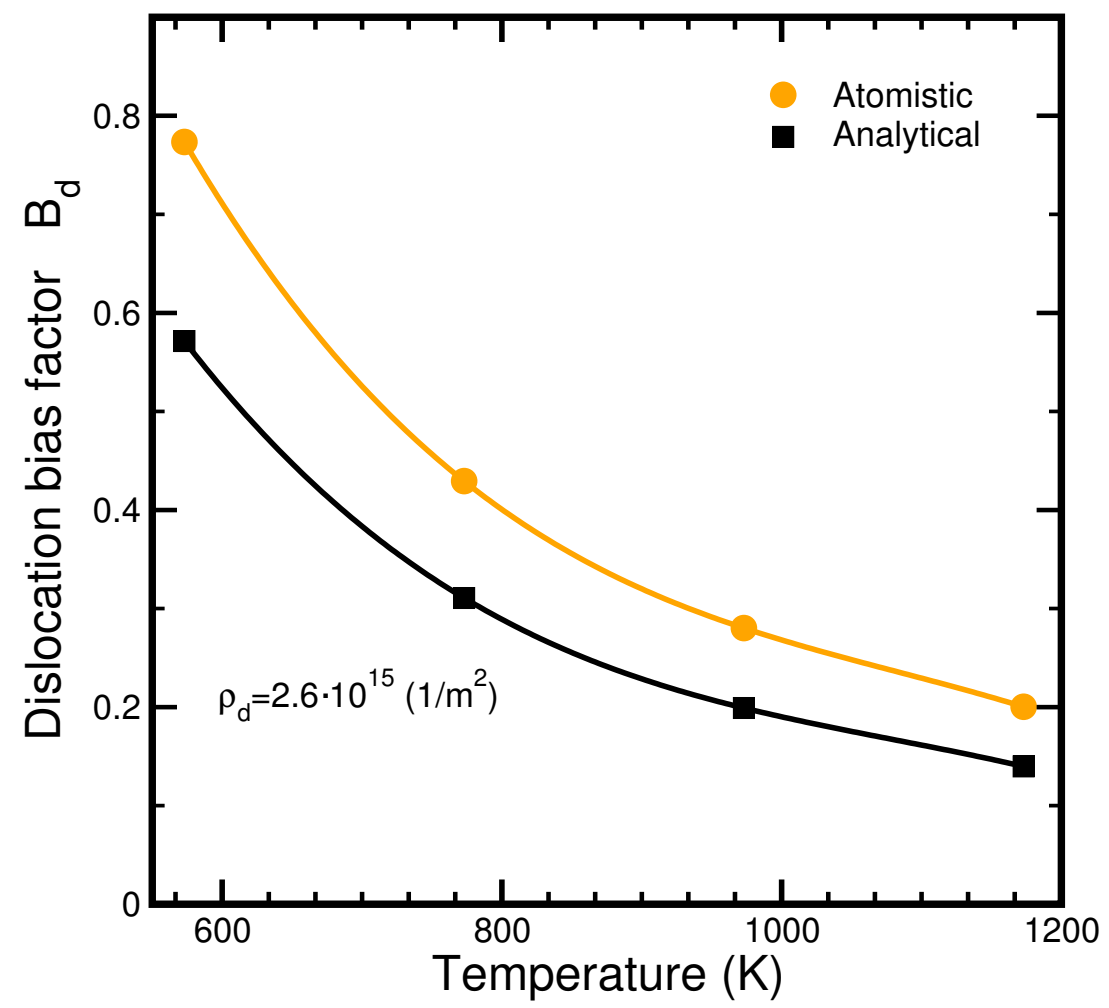

Figure 3: Temperature dependence of $B_{\mathrm{d}}$ for the atomistic and analytical cases. 


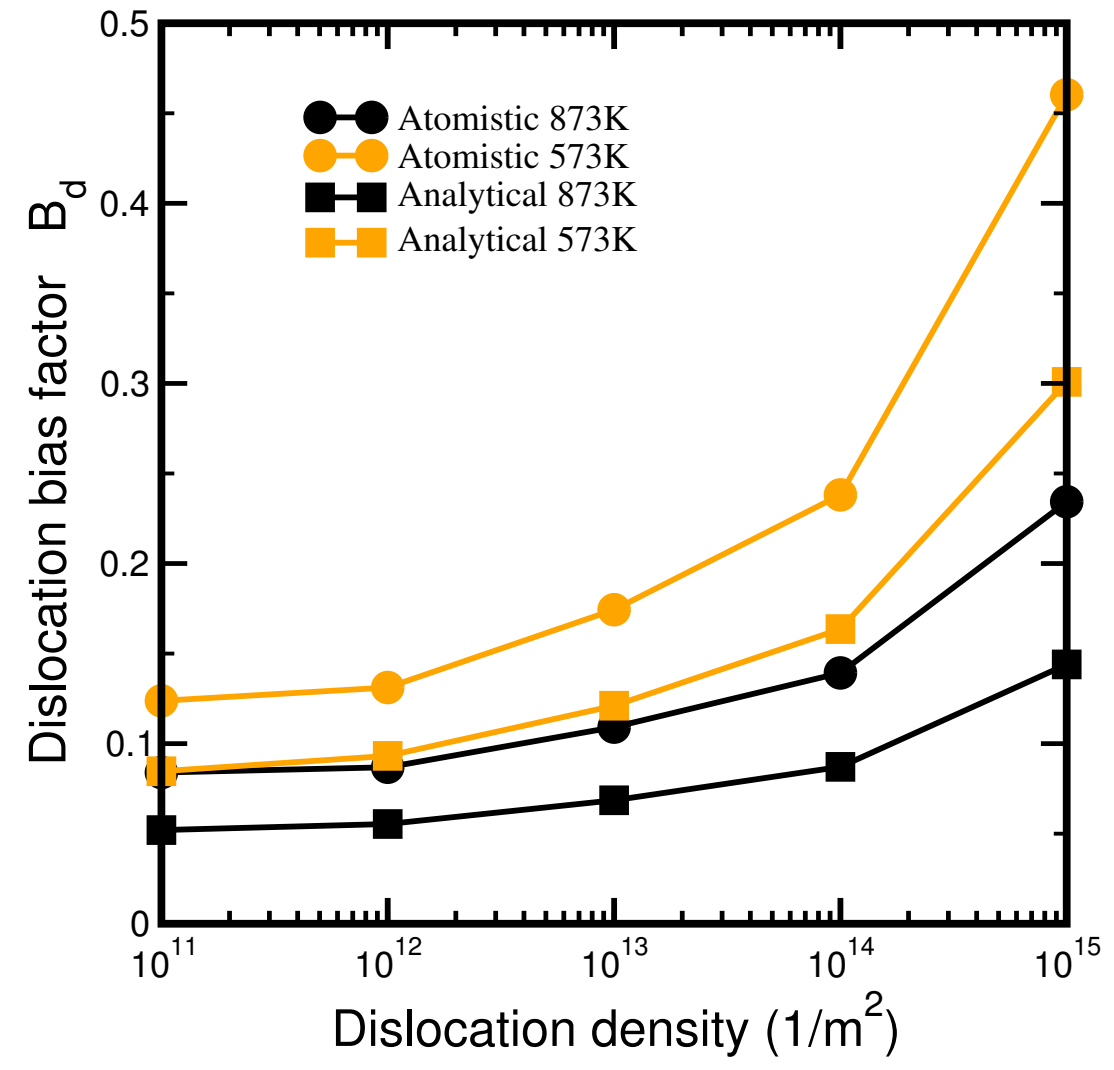

Figure 4: Dislocation density dependence of $B_{\mathrm{d}}$ for the atomistic and analytical cases at two temperatures. 
Table 1: SIA $\left(Z_{i}^{d}\right)$ and vacancy $\left(Z_{v}^{d}\right)$ sink strengths calculated at $873 \mathrm{~K}$ and a dislocation density of $2.6 \cdot 10^{15} \mathrm{~m}^{-2}$.

\begin{tabular}{c|ccc}
\hline & $Z_{\mathrm{i}}^{\mathrm{d}}$ & $Z_{\mathrm{v}}^{\mathrm{d}}$ & $B_{\mathrm{d}}$ \\
\hline \hline Atomistic & 1.34 & 1.00 & 0.34 \\
Analytical & 1.25 & 1.00 & 0.25 \\
\hline
\end{tabular}


Table 2: Experimental swelling induced by electron- ( $1^{\text {st }}$ row $)$ and proton $\left(2^{\text {nd }}, 3^{\text {rd }}\right.$ rows $)$ irradiation compared to steady state evaluation using the atomistic approach.

\begin{tabular}{c|cc}
\hline dose (dpa) & Reference swelling (\%) [?] & Calculated swelling (\%) \\
\hline \hline 0.013 & $0.2-1 \cdot 10^{-3}$ & $6.7 \cdot 10^{-3}$ \\
\hline 0.002 & $2.8 \cdot 10^{-4}$ & $5.3 \cdot 10^{-4}$ \\
0.008 & $1.9 \cdot 10^{-3}$ & $0.95 \cdot 10^{-3}$ \\
\hline
\end{tabular}

